\title{
Actualización \\ Recomendaciones para el rastreo y tratamiento de la hiperhomocisteinemia
}

\section{Introducción}

Dado que la enfermedad cardiovascular es la primera causa de muerte en Occidente y que muchas muertes son prevenibles, el hallazgo de nuevos factores de riesgo es de gran importancia. Respecto de la homocisteína, podemos decir que es una sustancia generada en el metabolismo de la metionina y que las personas con homocistinuria, un desorden autosómico recesivo, tienen hiperhomocisteinemia severa y enfermedad ateroesclerótica prematura. Se han descripto numerosos efectos de la homocisteína que corroboran la plausibilidad biológica como factor de riesgo vascular1, 2, 3. Las alteraciones en el metabolismo de la homocisteína han concentrado la atención por su rol potencial en la patogénesis de la enfermedad aterotrombótica ${ }^{4}$

\section{Asociación entre la homocisteína y el riesgo de eventos cardiovasculares}

La prevalencia de hiperhomocisteinemia en la población general es del 5 al 10\% (según tomemos como umbral, respectivamente, el percentilo 90 ó el 95)*. La tasa puede llegar al 30 o $40 \%$ en ancianos y, si los estudios epidemiológicos son correctos, hasta un $10 \%$ de los eventos coronarios podrían atribuirse a hiperhomocisteinemia $4,5,6$

\section{Pruebas para el rastreo de la hiperhomocis- teinemia}

La mayoría de las pruebas miden los niveles totales de homocisteína en ayunas ó bien cuatro a seis horas después de una carga oral con metionina $(0.1 \mathrm{mg} / \mathrm{kg})^{7,8}$. Las muestras deben ser conservadas en hielo para evitar el aumento espurio de la concentración de la homocisteína. Si el paciente se encuentra cursando alguna enfermedad aguda los resultados pueden ser falsamente bajos. El costo de las pruebas en Argentina oscila entre $\$ 30$ y $\$ 50$.

\section{Resumen de la evidencia y justificación de las recomendaciones}

Discutiremos en este artículo qué recomendaciones existen para la valoración de la homocisteinemia como estrategia en la evaluación de la enfermedad aterotrombótica (enfermedad coronaria, cerebrovascular, vascular periférica, trombosis venosa profunda, tromboembolismo pulmonar y eventos tromboembólicos inexplicados) en pacientes mayores de 17 años. Este debate surge ya que el rastreo podría identificar personas de alto riesgo en quienes se justifique llevar adelante un tratamiento agresivo de los factores de riesgo conocidos (ej. dislipemia) y/o nuevos como el que estamos analizando en este artículo.

El primer punto que queremos mencionar es que no existe consenso en la literatura acerca de a qué pacientes habría que dosarles homocisteína. Una aproximación razonable podría ser determinar homocisteína en pacientes de alto riesgo (historia familiar fuerte o enfermedad prematura) particularmente en ausencia de otros factores de riesgo. No obstante, las recomendaciones para el rastreo y tratamiento de la hiperhomocisteinemia en la población general deberán aguardar los resultados de los ensayos que están en curso.

Existen estudios en marcha que evaluarán si el tratamiento para reducir los valores de homocisteína reduce el riesgo de eventos vasculares. Dado la ausencia de evidencia grado I *, la Fuerza de Tareas Canadiense para Cuidados Preventivos, no recomienda el rastreo universal9. Sin embargo, la American Heart Association respalda el rastreo en poblaciones de alto riesgo y varios expertos avalan el tratamiento en grupos de alto riesgo.

\section{Metas de tratamiento}

Respecto de las metas del tratamiento, no existen guías basadas en la evidencia que las especifiquen. Una estrategia es emplear como referencia el percentilo 80 como umbral. Se aclara que el punto de corte puede variar de laboratorio en laboratorio.

\section{El valor de las dietas}

Las dietas ricas en ácido fólico han mostrado una serie de beneficios que incluyen la disminución del riesgo de defectos del cierre del tubo neural y de cáncer. Los pacientes deben ser estimulados para realizar este tipo de dietas por sus múltiples beneficios. Sin embargo, pareciera que la dieta rica en ácido fólico sin suplementos vitamínicos, es insuficiente para reducir los niveles de homocisteína ${ }^{10}$. Por otro lado, la suplementación con vitaminas en personas sanas con dietas variadas, no está recomendada.

De todas maneras, tampoco existe toxicidad ni contraindicación conocida para el uso de suplementos con ácido fólico y vitamina B12 (excepto en casos de anemia perniciosa)

\section{Estudios retrospectivos}

Hay 30 estudios de casos y controles que compararon los niveles de homocisteína de pacientes con enfermedad coronaria y los de sujetos sanos:

Luego de ajustar por los otros factores de riesgo, los pacientes con enfermedad coronaria presentaron niveles más altos de homocis teína en 22 de 27 estudios (OR: 1.2 a 10.9)11,12, 13, 14, 15, 16, 17. Metaanálisis de estudios retrospectivos confirmaron estos hallazgos. La relación entre los niveles de homocisteína y el número de vasos ocluídos no fue consistente.

La homocisteína puede estar relacionada con otros factores de riesgo como el tabaquismo, la hipertensión y la hiperglucemia. Sin embargo parece tener un efecto independiente y puede interactuar con otros factores de riesgo vascular.

\section{Estudios prospectivos}

Ocho estudios de casos y controles anidados evaluaron la asociación entre homocisteinemia y la ocurrencia de un primer evento cardiovascular mayor o el comienzo de una angina de pecho que necesitara revascularización. Sus resultados fueron conflictivos.

Los estudios de cohortes ${ }^{\star} 4,8,11,17$ sugieren que la hiperhomocisteinemia sería un factor de riesgo para eventos vasculares en pacientes con enfermedad coronaria establecida ya que en dos estudios se evidenciaron más eventos en los varones con hiperhomocisteinemia que en quienes no la manifestaban (el ajuste por la presencia de cardiopatía isquémica basal atenuó esta asociación) Niveles altos de homocisteína se relacionaron de manera independiente con la muerte por causa cardiovascular (RR 1.52 IC $95 \% 1.16$ a 1.98) en personas mayores de la cohorte de Framingham 4 .

La evidencia más contundente proviene del estudio de Nygard15 y colaboradores quienes siguieron prospectivamente a 587 pacientes con estenosis de las arterias coronarias documentada angiográficamente. Se observó una relación de dosis-respuesta entre los niveles basales de homocisteína y la mortalidad cardiovascular y global. Hallazgos similares se encontraron en pacientes con otras localizaciones de ateroesclerosis.

La evidencia proveniente de estudios prospectivos sugiere que la 
homocisteína actúa promoviendo la aparición de eventos agudos. Pocos estudios evaluaron su papel en la progresión de la ateroesclerosis.

En el Shunt Occlusion Trial, la oclusión al año del graft luego de la revascularización no se relacionó con los niveles de homocisteína.

En síntesis: la evidencia sugiere fuertemente que los niveles de homocisteína son un factor de riesgo para eventos agudos en pacientes con enfermedad vascular subyacente ${ }^{16}$. Sin embargo, dos estudios recientemente publicados de la cohorte de Caerphilly 17 18 (Gales) con 10 años de seguimiento no pudieron demostrar asociación entre niveles de homocisteína y eventos cardiovasculares ni cerebro vasculares.

\section{Asociación entre el folato sérico, los niveles de vitamina B6 y B12 y el riesgo de eventos cardiovasculares}

Varios estudios encontraron una relación inversa entre el nivel de folato sérico y los eventos cardiovasculares en el análisis univariado. Al ajustar por el nivel de homocisteína, esta asociación desaparece. Los niveles de B12 no se relacionan con enfermedad cardiovascular, sin embargo la vitamina B6 puede ser un factor independiente. Robinson y col. encontraron un mayor riesgo de enfermedad cardiovascular entre los pacientes con bajos niveles de B6 (OR 1.84 IC 95\% 1.32 a 2.42) que persistió luego del ajuste por niveles de homocisteína. Recíprocamente, el nivel de homocisteína es un predictor de enfermedad coronaria luego del ajuste por niveles de vitamina séricos.

\section{Efecto del tratamiento con vitaminas}

Varios estudios aleatorizados han evaluado el efecto de suplementos multivitamínicos en los niveles de homocisteína. Los regímenes que incluyen ácido fólico, B12 y B6 disminuyen los niveles séricos de la homocisteína entre un 20 y un $50 \%$. Un metanálisis de 12 estudios demostró que el ácido fólico disminuye la homocisteinemia en un $25 \%$. El agregado de vitamina B12, pero no de $\mathrm{B} 6$ reduce la homocisteinemia un $7 \%$ más. El efecto del ácido fólico fue máximo a una dosis de $0.5 \mathrm{mg} / \mathrm{día}$, sin embargo dosis de $0.2 \mathrm{mg} /$ día podrían ser suficientes. Dosis de $0.1 \mathrm{mg} / \mathrm{dí}$ a son inefectivas.

A pesar de que la vitamina B6 tiene poco efecto en los niveles basales de homocisteína, estudios no controlados han mostrado su utilidad en disminuir la hiperhomocisteienmia luego de una carga con metionina. Los niveles de homocisteína luego de la carga disminuyen entre un 31 y un $42 \%$ con B6.

En los pacientes que responden pobremente a la vitamina B6, el agregado de ácido fólico resulta eficaz. En la mayoría de los pacientes, seis semanas de tratamiento son suficientes para normalizar los niveles de homocisteína.

La recomendación de la FDA en los Estados Unidos de reforzar los derivados de los granos de cereales con 140 microgramos de ácido fólico cada $100 \mathrm{~g}$ de producto es insuficiente para normalizar niveles altos de homocisteína.

\section{Prevención de eventos cardiovasculares}

Todavía no sabemos si la disminución de los niveles de homocisteína será capaz de prevenir eventos cardiovasculares ya que no existen resultados de ensayos aleatorizados a largo plazo.

Existe cierta evidencia observacional que sugiere que el consumo de ácido fólico y vitamina B6 podría influir en la ocurrencia de eventos cardiovasculares. El Nurses'Health Study encontró que la mujeres que consumían más de 400 microgramos de ácido fólico por día o $3 \mathrm{mg}$ de vitamina $\mathrm{B} 6$ tenían menor riesgo de eventos $\mathrm{V}$ asculares ( $R R$ ajustado 0.69 para folato y 0.67 para vitamina $B 6$ ). Por otro lado, un estudio no controlado mostró regresión de placas carotídeas en 38 pacientes que recibieron suplementos de folato y vitamina $B 6$.

\section{Agenda de investigación}

Se encuentran en marcha ensayos aleatorizados a gran escala para evaluar la eficacia del tratamiento de la hiperhomocisteinemia en la prevención de eventos vasculares.

Todavía debe aclararse la dosis óptima de vitaminas y el papel de la carga con metionina en el diagnóstico de este desorden. Probablemente, habrá que revisar las políticas de fortificación de los alimentos.

La tabla 1 resume la evidencia existente sobre el tema.

Tabla 1: resumen de la evidencia

\begin{tabular}{|c|c|c|c|c|}
\hline \multicolumn{2}{|l|}{ Intervención } & Efectividad & $\begin{array}{l}\text { Grado de } \\
\text { evideneia }\end{array}$ & $\begin{array}{l}\text { Fuerza de la } \\
\text { recomendación }\end{array}$ \\
\hline \multirow[t]{2}{*}{$\begin{array}{l}\text { Rastreo de } \\
\text { hiper- } \\
\text { homocisteinemia }\end{array}$} & $\begin{array}{l}\text { Población } \\
\text { general }\end{array}$ & $\begin{array}{l}\text { Evidencia de asociación } \\
\text { entre los niveles plasmáti- } \\
\text { cos de homocisteína y la } \\
\text { enfermedad vascular. } \\
\text { No se conoce el impacto } \\
\text { del rastreo poblacional en } \\
\text { la disminución de eventos } \\
\text { vasculares. }\end{array}$ & \begin{tabular}{|l} 
Estudios de \\
cohortes y de \\
casos y \\
controles \\
$(\|-2)$
\end{tabular} & $\begin{array}{l}\text { Evidencia insuficiente } \\
\text { para recomendar o } \\
\text { desaconsejar el } \\
\text { rastreo o evidencia } \\
\text { grado C. }\end{array}$ \\
\hline & $\begin{array}{l}\text { Población } \\
\text { de alto } \\
\text { riesgo } \\
\text { vascular }\end{array}$ & $\begin{array}{l}\text { Estudios prospectivos } \\
\text { mostraron relación más } \\
\text { consistente entre los nive- } \\
\text { les de homocisteína y los } \\
\text { eventos vasculares en } \\
\text { pacientes con enfermedad } \\
\text { coronaria establecida. }\end{array}$ & & $\begin{array}{l}\text { Evidencia insuficiente } \\
\text { para recomendar o } \\
\text { desaconsejar el } \\
\text { rastreo o evidencia } \\
\text { grado C (@) }\end{array}$ \\
\hline \multicolumn{2}{|c|}{ Tratamiento con vitaminas } & $\begin{array}{l}\text { El tratamiento con ácido } \\
\text { fólico (sólo o con vitamina } \\
\text { B12) disminuye los nive- } \\
\text { les de homocisteína. La } \\
\text { vitamina B6 disminuye los } \\
\text { niveles luego de una carga } \\
\text { oral con metionina. }\end{array}$ & $\begin{array}{l}\text { Ensayos } \\
\text { aleatorizados } \\
\text { (I), estudios } \\
\text { de cohortes } \\
\text { (ll-2), y } \\
\text { estudios no } \\
\text { controlados. }\end{array}$ & $\begin{array}{l}\text { Evidencia insuficiente } \\
\text { para recomendar o } \\
\text { desaconsejar el } \\
\text { tratamiento o } \\
\text { evidencia grado C } \\
(\#) \text {. }\end{array}$ \\
\hline
\end{tabular}

(@) El rastreo podría identificar a personas de alto en quienes intensificar el tratamiento de los factores de riesgo conocidos. Por el momento existe evidencia insuficiente para rastrear con el propósito de tratar la hiperhomocisteinemia.

(\#) A pesar de que el ácido fólico disminuye los niveles de homocisteina plasmática existe insuficiente evidencia para afirmar que esto prevenga eventos vasculares.

\section{Conclusiones}

\section{Rastreo poblacional}

Por el momento, no está indicada la medición rutinaria de homocisteína plasmática en la población general ya que existe evidencia insuficiente para recomendar o desaconsejar el rastreo (Grado $\mathrm{C}$ ). Existe evidencia insuficiente para rastrear con el propósito de tratar la hiperhomocisteinemia.

Si bien el tratamiento con ácido fólico (sólo o con vitamina B12) disminuye los niveles de homocisteína, mientras que la vitamina B6 disminuye los niveles luego de una carga oral con metionina; no hay ensayos que hayan demostrado resultados clínicamente relevantes.

\section{Circunstancias en las que puede considerarse el dosaje homocisteína}

- Enfermedad prematura aterotrombótica (mujeres antes de los 65 y hombres antes de los 55 años) o severa (enfermedad en más de un territorio arterial o progresión rápida) sin factores de riesgo mayores (tabaquismo, hipetensión arterial, dislipemia y diabetes). 
- Trombosis venosa profunda o tromboembolismo de pulmón inexplicados o recurrentes.

- Un familiar en primer grado con enfermedad aterotrombótica prematura sin factores de riesgo mayores

- Un familiar de primer grado con hiperhomocisteinemia conocida.

\section{El redosaje no está indicado en pacientes con:}

- Una determinación previa normal si no cambia la condición clínica

- Valores anormales sin tratamiento

- Homocisteína en meta de tratamiento.

\section{Para llevar al consultorio}

Puede considerarse el dosaje homocisteína en los pacientes con:

- Enfermedad aterotrombótica severa prematura.o en ausencia de factores de riesgo mayores

- Trombosis venosa profunda o tromboembolismo de pulmón inexplicados o recurrentes.

- Un familiar de primer grado con enfermedad aterotrombóti ca prematura sin factores de riesgo mayores.

- Un familiar de primer grado con hiperhomocisteinemia conocida.

Dra. Karin Kopitowski [ Unidad de Medicina Famliar y Preventiva del Hospital Italiano de Buenos Aires ]

Dr. José Navarro Estrada [ Servicio de Cardiología. Hospital Italiano de Buenos Aires ]

\section{Bibliografía}

1. Ray JG.. Meta-analysis of hyperhomocysteinemia as a risk factor for venous thromboembolic disease. Arch Intern Med 1998; 158: 2101-6.

2. Moghadasian MH, Mc Manus BM, Frohlich JJ. Homocysteine and coronary artery disease. Clinical evidence and genetic and metabolic background. Arch Intern Med 1997; 157: 2299308.

3. Boushey CJ, Beresford SAA, Omenn GS, et al. A quantitive assesment of plasma homocysteine as a risk factor for vascular disease. Probable benefits of increasing folic acid intakes JAMA 1995; 274: 1049-57.

4. Bostom A, Silbershatz H, Rosenberg IH, et al. Nonfasting plasma total homocysteine levels and all-cause and cardiovascular disease mortality in eldery Framingham men and women Arch Intern med 1999; 159: 1077-80.

5. Eikelboom JW, Lonn E, Genest J Jr, et al. Homocysteine and cardiovascular disease: a critical review of epidemiological evidence. Ann Intern Med 1999; 131: $363-75$.

6. Cleophas $\mathrm{J}$, et al. Homocysteine, aris factor for coronary heart disease or not? A meta-analysis. Am J Cardiol; 2000: 86 1005-9.

7. Claph

7. Jeland $W$,

8. Egerton W, Silberberg J, Crooks R, et al. Serial measures of plasma homocysteine after acute myocardial infarction. Am 9. Booth GL, Wang EEL with the Canadian Task Force on Prevention for artery disease events. CMAJ 2000; 63(1):21- 29.
vention

vention for artery disease events. CMAJ 2000; 63(1) : 21- 29 .
10. Homocysteine Lowering Trialist' Collaboration. Lowering blood homocysteine with folic acid based supplements: a meta-analysis of randomised trials. BMJ 1998; $316: 894-8$.

10. Homocysteine Lowering Trialist' Collaboration. Lowering blood homocysteine with folic acid based supplements: a meta-analysis of randomised trials. BMJ 1998; $316: 894-8$.

11. Wald NJ, Watt HC, Law MR, et al. Homocysteine and ischemic heart disease. Results of prospective study with implications regarding prevention. Arch Intern med 1998; $158: 862-7$.
12. Bots ML, Launer LJ, Lindermans J, et al. Homocysteine and short-term risk of myocardial infarction and stroke in eldery. The Rotterdam study. Arch Intern med 1999; $159: 38-44$.

13. Evans RW, Shaten J. Hempel JD, et al. , for theMRFIT Research Group. Homocsteine and risk of cardiovascular disease in the MRFIT. Arteriosc Thromb Vasc Biol 1997; 17: 1947-53.

14. Verohef P, hennekens $\mathrm{CH}$, Allen RH, et al. Plasma total homocysteine and risk of angina pectoris with subsecuent coronary artery bypass surgery. Am J Cardiol 1997; 79: 799-01.

15. Nygard O. Nordrehaug JE, Refsum H, et al. Plasma homocysteine levels and mortality in patients with coronary artery disease. N Eng J Med 1997; $337: 230-6$.

16. Christen WG Ajani UA, Glynn RJ, Hennekens $\mathrm{CH}$ et al. Blood levels of homocysteine and increase risks of cardiovascular disease: causal or casual? Arch Intern Med 2000; 160(4): 16. Chisten

17. Fallon UB, Ben-Schlomo Y Elwood J. Homocysteine and coronary Herat disease in the Caerphilly cohort: a 10 year follow-up. Heart 2000; 85: 153-158.

18. Fallon UB, Ben-Schlomo Y, Elwood J. Homocysteine and ischemic stroke in men: the Caerphilly study. J Epidemiol Community health 2001; 55: 91-96.

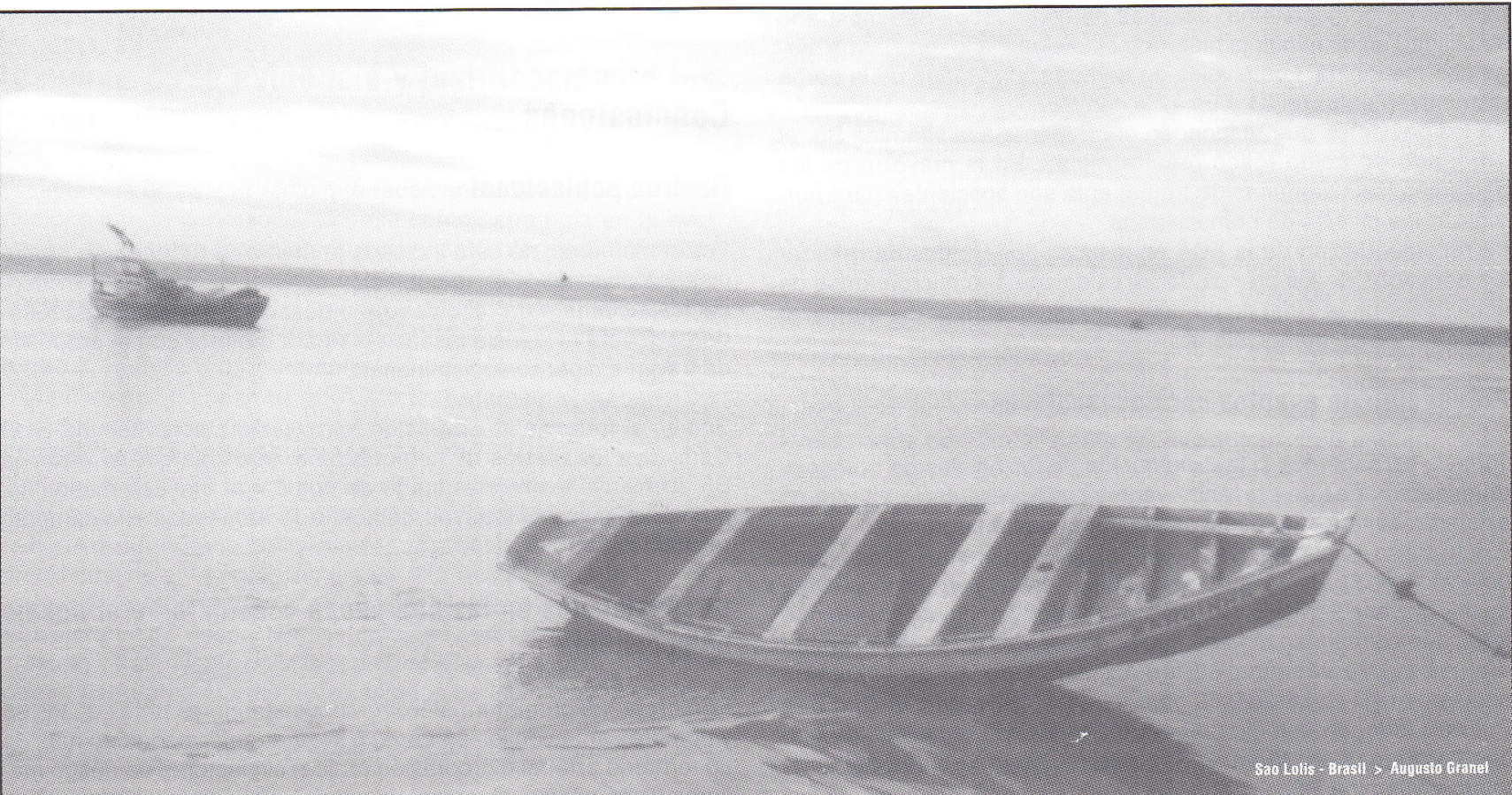

